Check for updates

Cite this: RSC Adv., 2019, 9, 20483

\title{
Study on the hypnotic effect of rare protopanaxadiol-type and protopanaxatriol-type ginsenosides $\dagger$
}

\author{
Ning Mou, ${ }^{a b c}$ Zhiguang Duan, ${ }^{a b c}$ Pei Ma, ${ }^{a b c}$ Rongzhan Fu*abc and Daidi Fan (D) *abc
}

Ginsenosides, as major active components of ginseng, possess various pharmacological activities, including anti-tumor, anti-diabetic and hypotensive effects. However, the sedative and hypnotic effect of ginsenosides and the involved mechanism remain unclear. In the present study, the hypnotic effect of rare protopanaxadiol-type (PD) ginsenosides, consisting of Rg3, Rk1, Rg5, and protopanaxatriol-type (PT) ginsenosides, consisting of Rh1, Rk3, Rh4, was investigated and compared in rodent models through behavioral pharmacology methods. Both rare PD and PT ginsenosides decreased spontaneous locomotion activity in normal mice and reduced sleep latency, and extended sleep duration in pentobarbital-treated mice. Moreover, PD and PT ginsenosides attenuated the insomnia induced by caffeine in mice. These hypnotic effects of PD and PT ginsenosides were potentiated by 5hydroxytryptophan (5-HTP), a precursor of serotonin, and inhibited by $p$-chlorophenylalanine (PCPA), a 5-HT synthesis inhibitor. Flumazenil (FLU, a specific gamma aminobutyric acid (GABA) antagonist) also impaired the hypnotic effect of both PD and PT ginsenosides. The aforementioned results indicated that PD and PT ginsenosides exhibit sedative and hypnotic activity, and PT ginsenosides show higher activity than PD ginsenosides at high doses $\left(96 \mathrm{mg} \mathrm{kg}^{-1}\right)$. Furthermore, the bioactivity of these two types of ginsenosides might be mediated via the serotonergic and GABAergic systems.

Received 1st March 2019 Accepted 26th May 2019 DOI: 10.1039/c9ra01549c rsc.li/rsc-advances
Ginseng (Radix Ginseng), famous for its high pharmacological activities, has been widely used as a restorative medicine in many East Asian countries for more than 2000 years. Ginseng saponins, known as ginsenosides, are the main components to which the pharmacological activities of ginseng are attributed. Since the first isolation of ginsenosides from $P$. ginseng in the 1960s, ${ }^{6}$ more than 150 natural ginsenosides have been identified from Panax species. ${ }^{7}$ Among them, major ginsenosides (ginsenosides Rb1, Rb2, Rc, Rd, and Rg1) comprise more than $80 \%$ of the total ginsenoside content of wild ginseng. Several major ginsenosides (ginsenosides Rb1, Rd, and Rg1) have been reported to regulate the central nervous system (CNS) and exert protective effects on neurodegeneration through studies in animals and neuronal cell culture ${ }^{8,9}$ In contrast to those major ginsenosides, the rare ginsenosides (ginsenosides Rg5, Rk1, Rk3, Rh4, F1, F2 and compound K), might be more effective drug candidates with higher pharmaceutical activity, due to their fewer sugar moieties and consequently greater readiness for cellular uptake. ${ }^{10}$ Therefore, the sedative-hypnotic effect and the underlying mechanism of rare ginsenosides must be investigated for the development of novel potent hypnotics with low side effects. However, the low concentration or absence of these rare ginsenosides in wild ginseng significantly hinders the exploration of their pharmaceutical activities and further clinical application. To date, the sedative and hypnotic effects of rare ginsenosides and the mechanisms involved have not yet 
been clarified. In previous studies, we successfully produced two types of rare ginsenoside mixtures biotransformed from major ginsenosides: protopanaxadiol-type (PD) and protopanaxatriol-type (PT) ginsenosides (the relevant data Fig. 1 can be found in $\mathrm{ESI} \dagger$ ), differentiated by the function group attached to the C6 position in aglycones whose type and number could greatly impact the biological activities of rare ginsenosides. These two types of rare ginsenosides have been proven to exert various pharmacological activities, such as cancer chemoprevention, ${ }^{\mathbf{1 1}}$ anti-inflammation ${ }^{\mathbf{1 2}}$ and antidiabetic effects. ${ }^{13}$ In the present study, we investigated and compared the sedative and hypnotic actions of rare PD and PT ginsenosides on normal mice and a model of insomnia mice, respectively, and subsequently proposed a potential underlying mechanism of these effects.

\section{Materials and methods}

\subsection{Animals}

The adult male Kunming mice (weighting $20 \pm 2 \mathrm{~g}$ ) used in this study were provided by the Experimental Animal Center of the Fourth Military Medical University (Xi'an, China). The mice were housed in an environmentally controlled room (constant temperature of $24-26^{\circ} \mathrm{C}$ and $55 \pm 5 \%$ humidity on a $12 \mathrm{~h}$ light/ dark cycle) and were given free access to diet and water during the study period. At week 1, the mice were given a new environment and normal diet. After the adaptation period, 60 mice were randomly divided into 6 groups. Each mouse was used for only one experiment. All animal procedures were performed in accordance with the Guidelines for Care and Use of Laboratory Animals of Northwest University, and approved by the Animal Ethics Committee of Northwest University.

\subsection{Drugs and materials}

2.2.1 Drugs. The rare ginsenosides used in this study were obtained from the laboratory through biotransformation using natural major ginsenosides as raw materials. 5-Hydroxytryptophan (5-HTP) and flumazenil (FLU) were purchased from Shanghai YuanYe Biological Technology Co., Ltd. p-Chlorophenylalanine (PCPA) and pentobarbital were purchased from Sigma (St Louis, Mo, United States). Caffeine was provided by Shanghai PureOne Biological Technology Co., Ltd. Diazepam (DZP) was acquired from Shandong Xinyi Pharmaceutical Co.,
Ltd. Medical grade polyethylene glycol (PEG) 400, was obtained from Jiangxi Yipusheng Pharmaceutical Co., Ltd. (Jiangxi, China).

2.2.2 High performance liquid chromatography (HPLC) analysis of PD and PT ginsenosides. PD and PT ginsenosides were converted from major ginsenosides Rb1 and Re by citric acid hydrolysis, respectively. Firstly, an appropriate amount of citric acid was added to the raw material for a $3 \mathrm{~h}$ reaction at 80$100{ }^{\circ} \mathrm{C}$ and $60-80{ }^{\circ} \mathrm{C}$, respectively. After adjusting, the $\mathrm{pH}$ to neutral, the PD and PT ginsenosides were obtain as precipitate through centrifugation, respectively. The precipitate was formulated into a $5 \mathrm{mg} \mathrm{ml}^{-1}$ solution with methanol for HPLC (SSI, United States) analysis using a C18 column ( $5 \mathrm{~mm}, 250 \mathrm{~mm}$ $\times 4.6 \mathrm{~mm}$, Omni Gene LLC) at $203 \mathrm{~nm}$. The gradient elution solvent included water and acetonitrile, at $50: 50$ and $60: 40$ for the production of PD and PT ginsenosides, respectively.

\subsection{Administration}

Sodium pentobarbital was dissolved in saline and injected intraperitoneally (i.p.) (0.1 ml/10 g). In this experiment, $48 \mathrm{mg}$ $\mathrm{kg}^{-1}$ (i.p.) sodium pentobarbital was used as the hypnotic dose with a $100 \%$ sleep rate, and $32 \mathrm{mg} \mathrm{kg}^{-1}$ (i.p.) was used as the subhypnotic dose with a $0 \%$ rate of sleep onset. Low-dose protopanaxadiol-type ginsenosides (LPD, $64 \mathrm{mg} \mathrm{kg}^{-1}$ ), highdose protopanaxadiol-type ginsenosides (HPD, $96 \mathrm{mg} \mathrm{kg}^{-1}$ ), low-dose protopanaxatriol-type ginsenosides (LPT, $64 \mathrm{mg} \mathrm{kg}^{-1}$ ), high-dose protopanaxatriol-type ginsenosides (HPT, $96 \mathrm{mg}$ $\left.\mathrm{kg}^{-1}\right)$ and diazepam $\left(2 \mathrm{mg} \mathrm{kg}^{-1}\right)$ were dissolved in a mixture of PEG400 and ultrapure water.

In our study, all drugs were given in a single dose. In the hypnotic dose pentobarbital-induced sleep test, LPD, HPD, LPT, HPT, and DZP were intragastrically administered $30 \mathrm{~min}$ before sodium pentobarbital administration (i.p.). The mice in the control group were given the same volume of solvent. At the end of the sodium pentobarbital injection, the sleep latency and sleep duration started to be recorded. In addition, animals that did not fall asleep within 15 min after administration of sodium pentobarbital were excluded from the experiments. ${ }^{\mathbf{1 4 - 1 6}}$ In the experiment of establishing an insomnia model with caffeine, caffeine was administered i.p. for $0.5 \mathrm{~h}$ before rare ginsenosides and DZP, the sodium pentobarbital was injected after $30 \mathrm{~min}$. In the synergistic experiment, 5 -HTP $\left(2.5 \mathrm{mg} \mathrm{kg}^{-1}\right)$ was given i.p. 15 min prior to the injection of sodium pentobarbital. In the
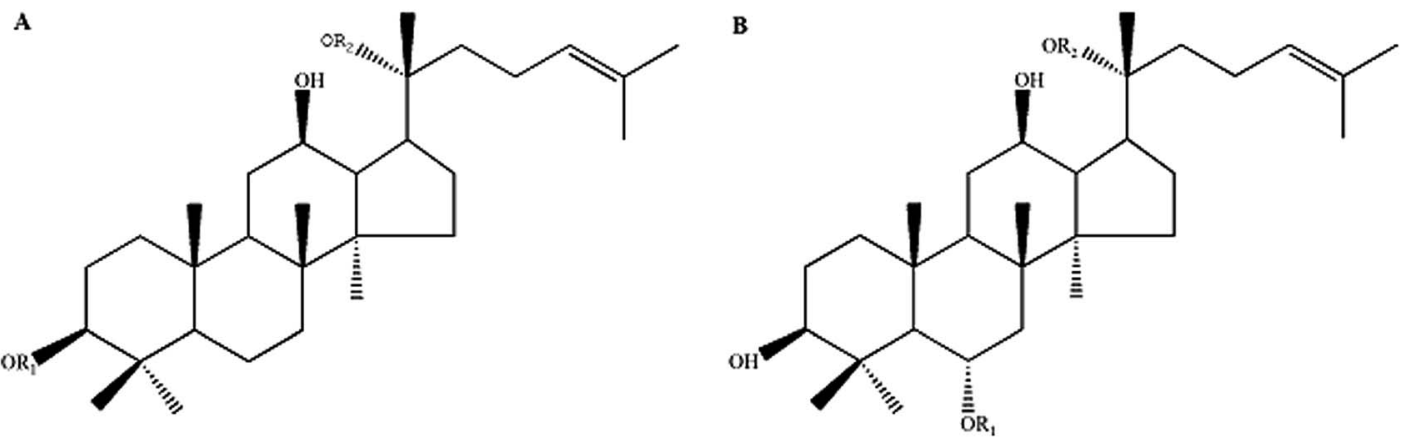

Fig. 1 The chemical molecular structure of protopanaxadiol-type ginsenosides (A) and protopanaxatriol-type ginsenosides (B). 
PCPA-induced insomnia experiment, LPD, HPD, LPT, HPT and DZP were administered $24 \mathrm{~h}$ after subcutaneous injection of PCPA (300 $\mathrm{mg} \mathrm{kg}^{-1}$ ). In the antagonist experiment, FLU (8 mg $\mathrm{kg}^{-1}$ ) was injected $15 \mathrm{~min}$ prior to the administration of LPD, HPD, LPT, HPT and DZP.

\subsection{Behavioral analyses}

2.4.1 Inner open-field behavior test. The mice were placed in a video-monitored open field (Chengdu Taimeng Software Co., Ltd., China) and adapted to the new environment for $5 \mathrm{~min}$. Then, the mice were administered LPD, HPD, LPT, HPT, and DZP. After $30 \mathrm{~min}$, the locomotion activity of the mice within $5 \mathrm{~min}$ was measured. At the end of each measurement, the enclosures were washed thoroughly with $75 \%$ alcohol to avoid effects on the next mouse. The locomotion time, locomotion distance, and the number of rearings were used as indicators for evaluating the sedative and hypnotic activity of rare ginsenosides.

2.4.2 Pentobarbital-induced sleep test. Experiments were performed between 8:30 and 11:30 a.m. After pentobarbital injection, the righting reflex disappeared over $1 \mathrm{~min}$, indicative of sleep. Sleep latency was recorded as the time elapsed between the administration of pentobarbital and the loss of the righting reflex. Sleep duration was recorded as the elapsed time from righting reflex loss to recovery.

\subsection{Statistics and analysis}

All data are expressed as the mean \pm standard error of mean (SEM). The experimental data were statistically analyzed by the SPSS 22.0 (SPSS Inc., Chicago, IL, United States) statistical program for Windows. For statistical comparison, the results were analyzed using GraphPad Prism software (Version 5.0, GraphPad Software Inc., San Diego, CA, United States) with oneway analysis of variance (ANOVA) followed by Tukey's post hoc analysis. For analysis of the subhypnotic dosage in the pentobarbital test, a chi-square test was used to compare the number of mice that fell asleep. A value of $p<0.05$ was regarded as statistically significant. $*, * *$, and $* * *$ are used to indicate $p<$ $0.05, p<0.01$, and $p<0.001$, respectively.

\section{Results}

\subsection{Identification of PD and PT ginsenosides}

An HPLC method was developed to analyze the main components of rare PD and PT ginsenosides (Fig. 2A and B, other relevant data can be found in ESI $\dagger$ ), and the ratios of the major components are shown in Table 1. PD ginsenosides mainly contained four components, namely, ginsenoside $S$-Rg3 $(28.57 \pm 0.27 \%), R$-Rg3 $(26.74 \pm 0.47 \%)$, Rk1 $(22.19 \pm 0.26 \%)$ and $\operatorname{Rg} 5(22.16 \pm 0.68 \%)$. Meanwhile, the four major components in PT ginsenosides were ginsenosides $S$-Rh1, $R$-Rh1, Rk3 and Rh4. Among them, the content of ginsenoside Rh4 $(49.08 \pm 0.26 \%)$ was the highest and accounted for almost half of the total PT ginsenoside content, while the contents of Rk3, $R$-Rh1 and $S$-Rh1 were $24.05 \pm 0.09 \%$, $16.44 \pm 0.56 \%$ and $9.11 \pm 0.53 \%$, respectively.

\subsection{The sedative effect of PD and PT ginsenosides in normal mice}

The sedative effect of PD and PT ginsenosides was first evaluated based on the spontaneous locomotion activity of mice measured by open-field experiments. The number of rearings in both the PD and PT groups was significantly lower than that in the vehicle group (Fig. 3) and was similar to that in the diazepam group ( $2 \mathrm{mg} \mathrm{kg}^{-1}$, i.g.). Only a high dose $\left(96 \mathrm{mg} \mathrm{kg}^{-1}\right.$, i.g.) of PD ginsenosides resulted in an effective decrease in locomotion time compared to vehicle $(p<0.05)$. In contrast, both low and high doses of PT ginsenosides reduced the locomotion time ( $p<0.05$, Fig. 4A). All doses of PD and PT ginsenosides similarly reduced locomotion distance compared with vehicle (Fig. 4B). These results suggest that both rare PD and PT ginsenosides have a significant sedative effect.

\subsection{Effects of PD and PT ginsenosides in normal mice treated with pentobarbital}

A subhypnotic dose (32 $\mathrm{mg} \mathrm{kg}^{-1}$, i.p.) and hypnotic dose $(48 \mathrm{mg}$ $\mathrm{kg}^{-1}$, i.p.) of pentobarbital were used to determine the effect of rare PD and PT ginsenosides on the incidence and duration of sleep in normal mice. HPD, LPT and HPT significantly increased the incidence of sleep in mice induced by the subhypnotic dose of sodium pentobarbital compared to vehicle (Table 2). When the hypnotic dose of sodium pentobarbital was used, HPD $(p<0.01)$, LPT $(p<0.05)$ and HPT $(p<0.05)$ effectively shortened the sleep latency of mice (Fig. 5A). In addition, HPD increased the sleep duration more than LPD $(p<0.001)$. However, LPT and HPT exerted similar prolonging effects on sleep duration (Fig. 5B). These results indicated that rare PD and PT ginsenosides increase sleep in normal mice, with PT ginsenosides showing higher activity than PD ginsenosides.

\subsection{Effects of PD and PT ginsenosides on insomnia induced by caffeine in mice}

To explore the efficacy of rare PD and PT ginsenosides in the treatment of insomnia, caffeine was injected $0.5 \mathrm{~h}$ before the gavage administration of rare ginsenosides to establish the insomnia model according to previous reports. ${ }^{17,18}$ Compared to caffeine alone, HPD, LPT, and HPT effectively attenuated caffeine-induced insomnia by reducing sleep latency (Fig. 6A) and prolonging sleep duration (Fig. 6B). In particular, the reduction in sleep latency caused by $\operatorname{HPD}(p<0.01)$ was stronger than that caused by LPD, whereas there was no difference between the effect of LPT and HPT. Although LPD had no influence on sleep latency, it effectively prolonged sleep duration $(p<0.001)$. The above results indicated that administration of rare PD and PT ginsenosides alleviated caffeine-induced insomnia in mice.

\subsection{Synergistic effect of PD and PT ginsenosides with 5-HTP on pentobarbital-induced sleep}

To investigate the relationship between the hypnotic effect of rare PD and PT ginsenosides and the serotonergic system, 15 min after the gavage administration of PD and PT 


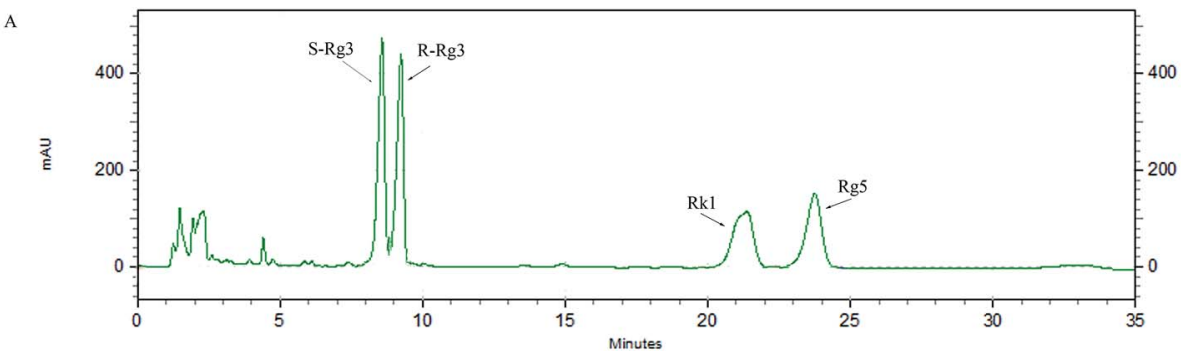

B

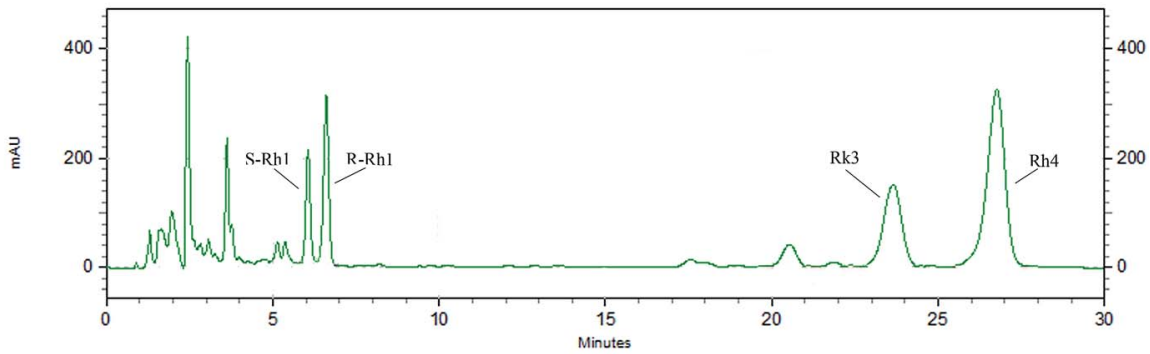

Fig. 2 HPLC chromatograms of PD (A) and PT (B).

Table 1 Composition and contents of PD and PT analyzed by HPLC

\begin{tabular}{llll}
\hline & Compounds & Retention time (min) & Percentage (\%) \\
\hline \multirow{2}{*}{ PD } & $S$-Rg3 & 8.58 & $28.57+0.27$ \\
& $R$-Rg3 & 9.24 & $26.74+0.47$ \\
& Rk1 & 21.37 & $22.19+0.26$ \\
& Rg5 & 23.75 & $22.16+0.68$ \\
PT & $S$-Rh1 & 6.06 & $9.11+0.53$ \\
& $R$-Rh1 & 6.60 & $16.44+0.56$ \\
& Rk3 & 23.64 & $24.05+0.09$ \\
& Rh4 & 26.76 & $49.08+0.26$
\end{tabular}

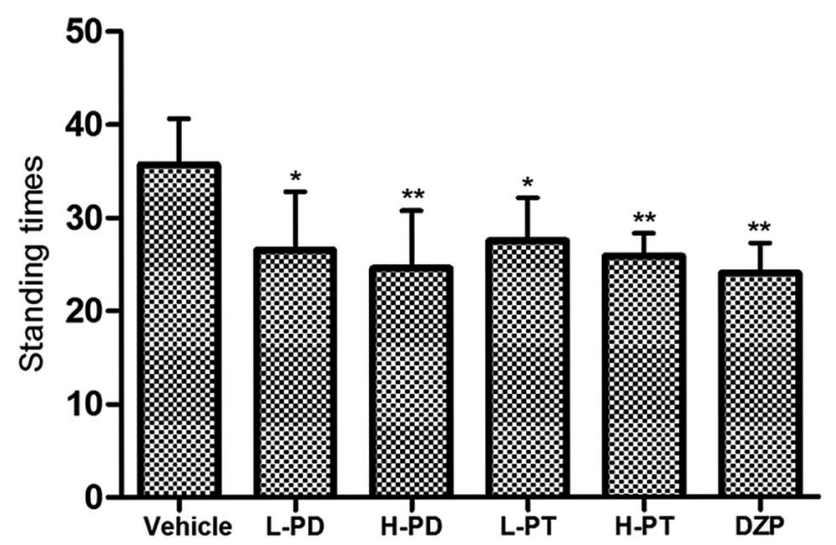

Fig. 3 Effects of ginsenosides on the standing times of pentobarbitaltreated mice $(n=10)$. All data are presented as mean + S.E.M., $* p<$ $0.05, * * p<0.01, * * * p<0.001$ vs. vehicle group.

ginsenosides, mice were injected with 5-HTP $\left(2.5 \mathrm{mg} \mathrm{kg}^{-1}\right)$, followed by sodium pentobarbital 15 min later. With 5-HTP injection alone, sodium pentobarbital $\left(32 \mathrm{mg} \mathrm{kg}^{-1}\right)$ was unable to induce sleep in mice. In contrast, after coadministration of 5-
HTP and ginsenosides, the rate of sleep onset in both the LPD ( $p$ $<0.05)$ and LPT $(p<0.01)$ groups was increased (Table 3$)$. In addition, coadministration of LPD and 5-HTP shortened the sleep latency ( $p<0.01)$, whereas coadministration of HPD and 5HTP not only shortened the sleep latency $(p<0.01)$ but also prolonged the sleep duration $(p<0.05)$ induced by sodium pentobarbital $\left(48 \mathrm{mg} \mathrm{kg}^{-1}\right)$ in mice. Only the high dose of PT ginsenosides in combination with 5-HTP shortened the latency $(p<0.05)$ and prolonged the sleep duration $(p<0.05)$, whereas the low dose did not show such effects (Fig. 7).

\subsection{Effects of PD and PT ginsenosides on PCPA-induced insomnia in mice treated with pentobarbital}

According to previous research, the use of $300 \mathrm{mg} \mathrm{kg}^{-1} \mathrm{p}$ chlorophenylalanine (PCPA) $24 \mathrm{~h}$ in advance can cause complete insomnia in mice, ${ }^{19}$ which was also confirmed in this experiment, showing a prolonged sleep latency $(p<0.001)$ and shorted sleep duration with PCPA treatment $(p<0.05)$ (Fig. 8). After the administration of ginsenosides, the effect of PCPA on insomnia was significantly attenuated. High doses of PD and PT ginsenosides had higher activity than low doses, which was shown in the shortened sleep latency ( $p<0.001$, Fig. 8A) and prolonged sleep duration in the HPD $(p<0.01)$ and HPT groups $(p<0.05)$ compared with those of the vehicle group given PCPA (Fig. 8B).

\subsection{Effects of PD and PT ginsenosides on the reversal of hypnosis induced by pentobarbital in mice treated with flumazenil}

Flumazenil (FLU) is an antagonist that inhibits the sedative and hypnotic activity of GABA-A-BZD agonists, such as DZP, by preventing their binding to the GABA-A receptor. In this study, mice were pretreated with flumazenil before ginsenoside administration, and sleep was then induced with sodium 
A

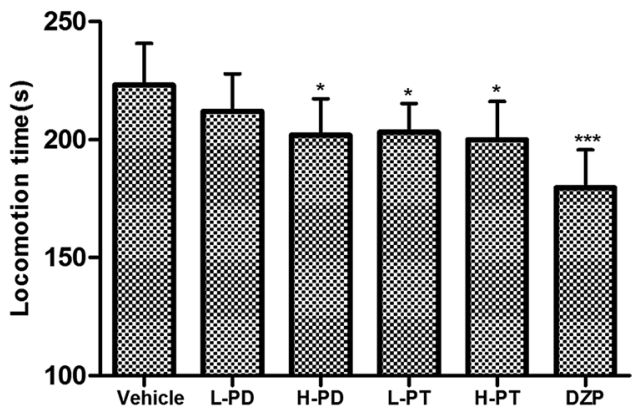

B

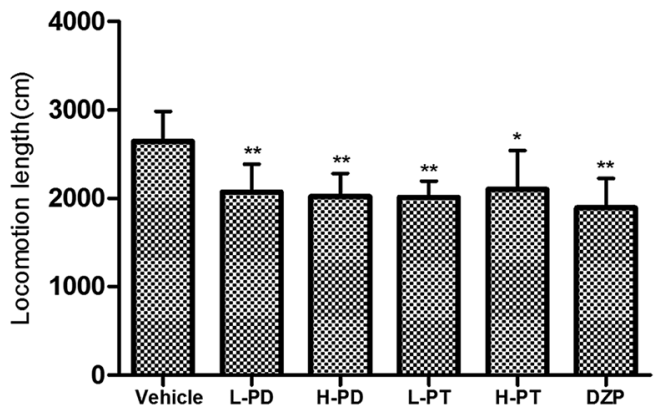

Fig. 4 Effects of ginsenosides on locomotion time (A) and locomotion length (B) in mice $(n=10)$.

Table 2 The effect of ginsenosides on the sleep onset of mice treated with sub-hypnotic dose of sodium pentobarbital. ${ }^{*} p<0.05, * * p<0.01$, $* * * p<0.001$ vs. vehicle group, chi-square test

\begin{tabular}{llll}
\hline Group & Dosage $\left(\mathrm{mg} \mathrm{kg}^{-1}\right)$ & $\begin{array}{l}\text { No. of falling } \\
\text { asleep/total }\end{array}$ & $\begin{array}{l}\text { Sleep onset } \\
(\%)\end{array}$ \\
\hline Vehicle & - & $0 / 10$ & 0 \\
LPD & 64 & $4 / 10$ & 40 \\
HPD & 96 & $6 / 10$ & $60^{*}$ \\
LPT & 64 & $7 / 10$ & $70^{* *}$ \\
HPT & 96 & $8 / 10$ & $80^{* *}$ \\
DZP & 2 & $10 / 10$ & $100^{* * *}$
\end{tabular}

pentobarbital to clarify the action of the GABAergic system on the hypnotic effect of rare PD and PT ginsenosides. FLU significantly attenuated the effect of DZP by increasing the sleep latency ( $p<0.01$, Fig. 9A) and decreasing the sleep duration $(p<$ 0.001, Fig. 9B). Similar to the effect of FLU on DZP, FLU diminished the LPD-, HPD-, and HPT-induced potentiation of pentobarbital-induced sleep (Fig. 9). Interestingly, FLU exerted a stronger attenuation of the hypnotic effect of PD ginsenosides than on that of PT ginsenosides.

\section{Discussion}

A wide range of pharmacological effects of rare ginsenosides have already been exhibited, including immune regulation, antioxidation, anti-inflammation, and anti-tumor effects, ${ }^{20-23}$ for which rare ginsenosides have already attracted increasing attention from many researchers.

However, the role of rare ginsenosides in the central nervous system has not been studied. Therefore, the sedative and hypnotic effects of rare PD and PT ginsenosides, and the underlying mechanism were investigated in the present study. Among the pharmacological methods for assessing sedative and hypnotic activity, the open-field test and the sodium pentobarbital-induced sleep test are the two classic behavioral methods. ${ }^{24}$ The locomotion activity measured in the open-field test is commonly considered an important indicator of alertness, and a decrease in spontaneous motor activity is often regarded as a sedative behavior. ${ }^{25}$ The present study showed that both low- and high-dose rare PD and PT ginsenosides reduced the number of rearings and the locomotion distance of normal mice. Low-dose PD ginsenosides did not influence the locomotion time of mice, but HPD, LPT and HPT showed significant inhibitory effects. Furthermore, sleep latency and sleep duration obtained in the sodium pentobarbital-induced sleep test are also commonly used as indicators for assessing the sedative and hypnotic effects of drugs. LPD only increased sleep duration but had no effect on sleep latency. HPD, LPT and HPT effectively shortened the sleep latency and prolonged the sleep duration of normal mice.

Caffeine, has been proven to have a marginal effect on the circadian rhythm of sleep-wake regulation in the human body. ${ }^{26}$ Previous studies reported that a low dosage of caffeine before bedtime prolongs sleep latency and affects sleep efficiency. ${ }^{27}$ Caffeine administration, hence, provides a simple, safe
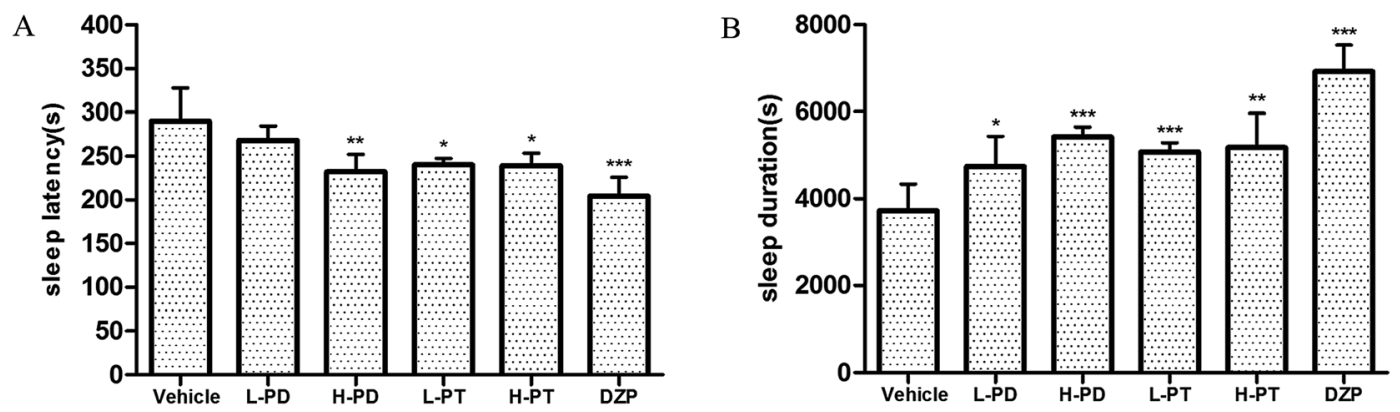

Fig. 5 Effects of ginsenosides on the hypnotic response to pentobarbital-induced sleep in normal mice. The sleep latency (A) and sleep duration (B) are assessed. All data are presented as mean + S.E.M. $(n=10), * p<0.05, * * p<0.01, * * * p<0.001$ vs. the vehicle mice. 

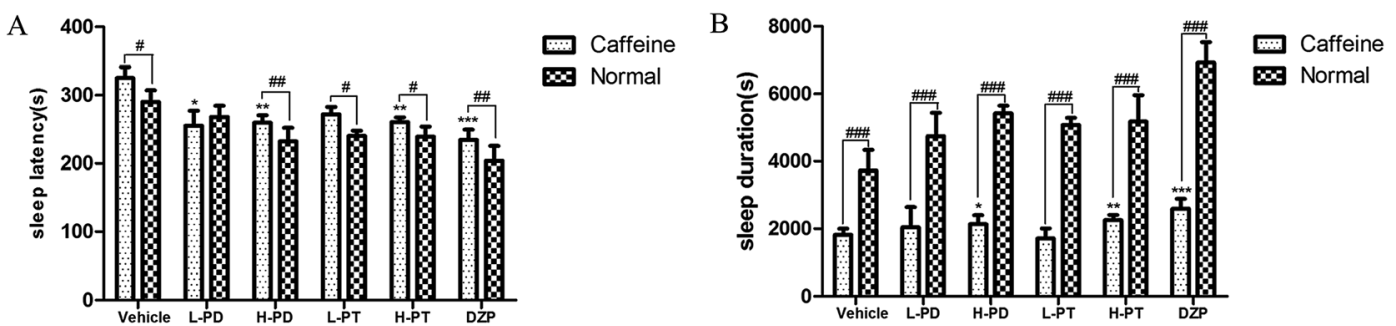

Fig. 6 Effects of ginsenosides on caffeine-induced insomnia in pentobarbital-treated mice $(n=10)$. The sleep latency (A) and sleep duration (B) are assessed. All data are presented as mean + S.E.M., ${ }^{*} p<0.05,{ }^{* *} p<0.01,{ }^{* * *} p<0.001$ vs. vehicle group, ${ }^{*} p<0.05,{ }^{\# \#} p<0.01,{ }^{\# \# \#} p<0.001$ vs. normal group.

Table 3 Synergic effect of ginsenosides with 5-HTP on sleep onset of mice treated with sub-hypnotic dosage of pentobarbital. *p $<0.05$, $* * p<0.01, * * * p<0.001$ vs. vehicle group, chi-square test

\begin{tabular}{llll}
\hline Group & Dosage $\left(\mathrm{mg} \mathrm{kg}^{-1}\right)$ & $\begin{array}{l}\text { No. of falling } \\
\text { asleep/total }\end{array}$ & $\begin{array}{l}\text { Sleep onset } \\
(\%)\end{array}$ \\
\hline Vehicle & - & $0 / 10$ & 0 \\
DZP & 2 & $10 / 10$ & $100^{* * * *}$ \\
5-HTP & 2.5 & $2 / 10$ & 20 \\
LPD & 64 & $4 / 10$ & 40 \\
LPD + 5-HTP & $64+2.5$ & $6 / 10$ & $60^{*}$ \\
LPT & 64 & $7 / 10$ & $70^{* *}$ \\
LPT + 5-HTP & $64+2.5$ & $8 / 10$ & $80^{* *}$ \\
\hline
\end{tabular}

and efficient method to reproduce the underlying feature of insomnia in animals and humans. In our study, HPD, LPT and HPT partially attenuated caffeine-induced insomnia in mice by shorting sleep latency and prolonging sleep duration (Fig. 6). Based on the above results, rare PD and PT ginsenosides can be inferred possess a hypnotic effect in normal mice, with PT ginsenosides having a slightly greater effect than PD ginsenosides.

The differentiation in the effectiveness of PD and PT ginsenosides may be largely attributed to the difference in their composition of ginsenoside. The main ginsenoside of PD and PT ginsenosides, which is responsible for almost half of the total content, is ginsenoside Rg3 (two sugar residues at C3) and ginsenoside Rh4 (one sugar residue at $\mathrm{C6}$ ), respectively. Thus, PT ginsenosides, which contain ginsenosides with a simpler structure, may exhibit stronger activity than PD ginsenosides, in agreement with previous studies showing that the pharmacological activity of ginsenosides, such as its anti-cancer activity, increases with a decrease in the number of sugar moieties in ginsenoside molecules. ${ }^{28-30}$

Serotonin, 5-hydroxytryptamine (5-HT), is a monoamine neurotransmitter, that contributes to feelings of well-being and happiness, improves sleep, and modulates cognition, memory, and numerous physiological processes. ${ }^{31-33}$ In particular, serotonin synthesized in serotonergic neurons of the CNS plays an important role in the regulation of mood, appetite and the sleep-wake cycle. ${ }^{34}$ Thus, modulation of serotonin is the major mechanism of several classes of hypnotics and pharmacological antidepressants. ${ }^{34}$ The precursor of serotonin, 5-hydroxytryptophan (5-HTP), enables an increase in the levels of 5-HT, consequently causing drowsiness and extending sleep duration. ${ }^{35}$ In contrast, PCPA (a 5-HT synthesis inhibitor) reduces sleep and induces insomnia, and has been widely used to establish insomnia models related to the serotonin system. The insomnia effect of PCPA can be reversed by treatment with 5HTP through restoring serotonin synthesis. ${ }^{36}$ To clarify the relationship between the serotonergic system and the hypnotic effect of rare ginsenosides, the influence of the PCPA/5-HTP model on the hypnotic effect of PD and PT ginsenosides was investigated. Our results suggested that both low-dose PD and low-dose PT ginsenosides exhibited synergistic effect with 5HTP, increasing the occurrence of sleep induced by the subhypnotic dose of sodium pentobarbital (Table 3). The sleep latency was reduced by the coadministration of 5-HTP and PD ginsenosides at different doses, and high doses of PT ginsenosides. In contrast, the sleep duration was prolonged by coadministration of 5-HTP with only a high dose of PD and PT ginsenosides (Fig. 7). In addition, both high-dose PD and high-
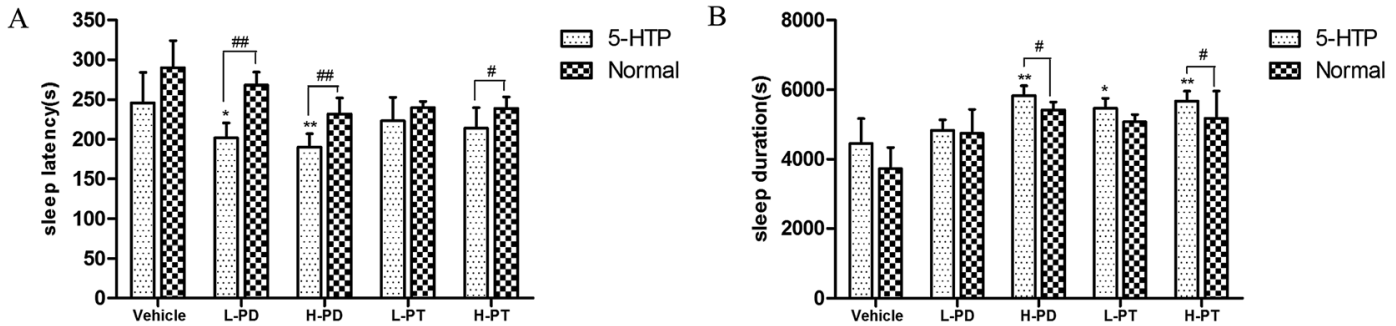

Fig. 7 Synergic effect of ginsenosides with 5-HTP on hypnotic response in pentobarbital treated mice $(n=10)$. The sleep latency (A) and sleep duration (B) are assessed. All data are presented as mean + S.E.M., ${ }^{*} p<0.05,{ }^{* *} p<0.01$ vs. vehicle group, ${ }^{*} p<0.05,{ }^{\# \#} p<0.01$ vs. normal group. 
A

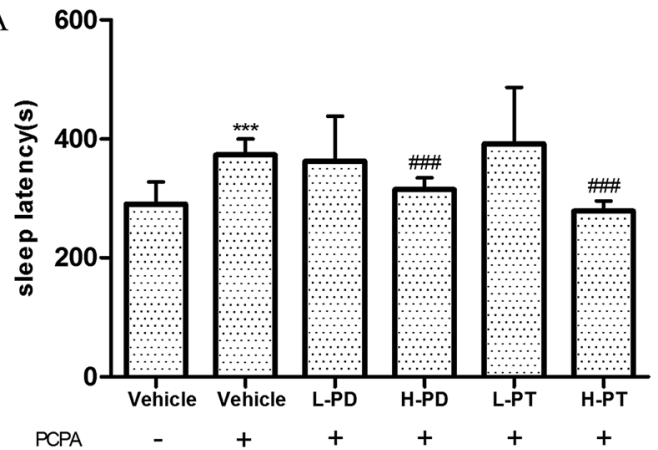

B

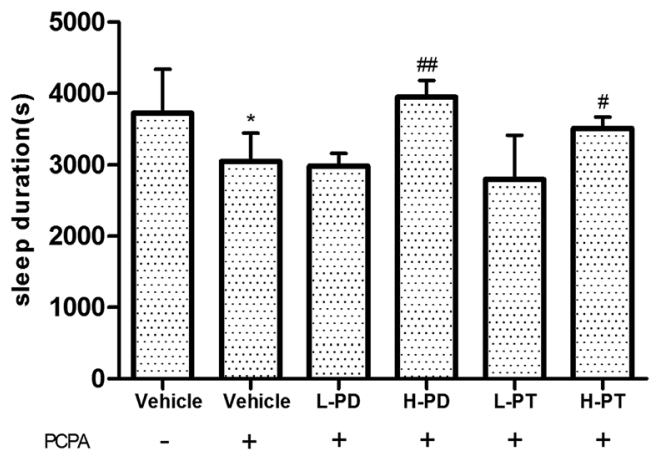

Fig. 8 Effects of ginsenosides on PCPA-induced insomnia in pentobarbital-treated mice $(n=10)$. The sleep latency (A) and sleep duration (B) are assessed. All data are presented as mean + S.E.M., ${ }^{*} p<0.05,{ }^{* * *} p<0.001$ vs. vehicle group without PCPA; ${ }^{\#} p<0.05,{ }^{\# \#} p<0.01$, ${ }^{\# \# \#} p<0.001$ vs. vehicle group with PCPA.

dose PT ginsenosides were capable of significantly attenuating the suppression effect of PCPA on the hypnotic effect of pentobarbital (Fig. 8). These results indicated that the serotonergic system was involved in the hypnotic effect of rare PD and PT ginsenosides, and there was no significant difference between the effect of PD and PT ginsenosides on the serotonergic system. Nevertheless, prior studies have revealed that different types of 5-HT receptors are involved in the serotonergic system to mediate both excitatory and inhibitory neurotransmission in the central and peripheral nervous systems. ${ }^{37,38}$ For example, a decline in neuronal activity induced by $5-\mathrm{HT}_{1 \mathrm{~A}}$ receptor-mediated choline arousal in the basal forebrain may be associated with sleep development. ${ }^{39,40}$ Although the hypnotic effects of PD and PT ginsenosides are related to the serotonergic system, their actions might be mediated by different 5-HT receptors, which need to be further studied in the future.

Gamma-aminobutyric acid (GABA), one of the major inhibitory neurotransmitters in the central nervous system, serves as a key signaling molecule modulating neuronal excitability. ${ }^{41}$ The bioactivity of GABA is mainly mediated by activating the $\mathrm{GABA}_{\mathrm{A}}$ receptor, a ligand-gated ion channel, to raise intracellular $\mathrm{Cl}^{-}$levels. In addition to the receptor site of its endogenous ligand $\mathrm{GABA}$, the $\mathrm{GABA}_{\mathrm{A}}$ receptor possesses a binding site for the benzodiazepine (BZD) class of tranquilizer drugs. Once bound to benzodiazepine, the $\mathrm{GABA}_{\mathrm{A}}$ receptor changes its conformation, showing a much higher affinity for GABA, leading to sedative and hypnotic effects. This benzodiazepine binding site on the $\mathrm{GABA}_{\mathrm{A}}$ receptor is the target of many hypnotics, including nonbenzodiazepine ' $\mathrm{Z}$ drugs' (zaleplon, zolpidem, zopiclone and eszopiclone). ${ }^{42}$ In this study, flumazenil (FLU), a GABA-A-BZD antagonist, was used to address the involvement of the GABAergic system in the sedative and hypnotic effects of rare PD and PT ginsenosides. FLU reversed the action of both low- and high-dose PD ginsenosides but only high-dose PT ginsenosides in pentobarbital-treated mice. This effect was found to be similar to that of magnolol, which is an active element of Magnolia officinalis, and effectively reversed by FLU for sleep-enhancing function..$^{42}$ Therefore, the hypnotic effect of rare PD and PT ginsenosides could be surmised to be delivered through the $\mathrm{GABA}_{\mathrm{A}}$ receptor. Moreover, the inhibitory effects of FLU on the hypnotic effect of rare PD ginsenosides were stronger than those on the hypnotic effect of rare PT ginsenosides, suggesting that PD ginsenosides might play a more important role in the GABAergic system than PT ginsenosides when exerting a sedative and hypnotic effect.

In summary, rare PD and PT ginsenosides are capable of delivering a sedative and hypnotic effect, and alleviating the symptoms of insomnia. The effective dosage range of PD ginsenosides is $64-96 \mathrm{mg} \mathrm{kg}^{-1}$, whereas PT ginsenosides exhibit a similar effect of improving sleep at both doses. In addition, the underlying mechanisms for the hypnotic effects of rare PD and PT ginsenosides were elucidated to be related to both the serotonergic system and the GABAergic system, and rare PD
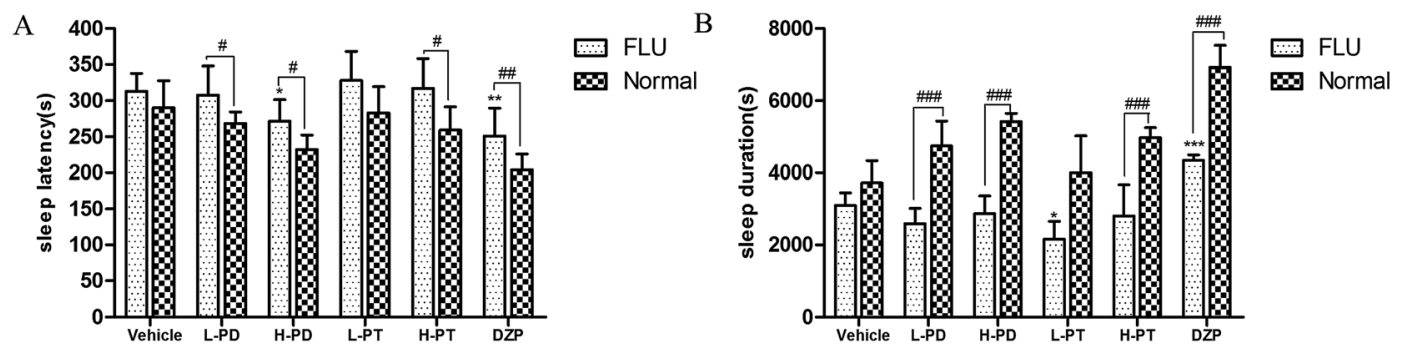

Fig. 9 Synergic effect of ginsenosides with FLU on hypnotic response in pentobarbital treated mice $(n=10)$. The sleep latency (A) and sleep duration (B) are assessed. All data are presented as mean + S.E.M., ${ }^{*} p<0.05, * * p<0.01, * * * p<0.001$ vs. vehicle group, ${ }^{\#} p<0.05,{ }^{\# \#} p<0.01,{ }^{\# \# \#} p$ $<0.001$ vs. normal group. 
ginsenosides might possess a stronger effect on the GABAergic system than PT ginsenosides. To our knowledge, this is the first study of the sedative and hypnotic effects of rare PD and PT ginsenosides and the possible mechanisms involved. Further studies are required to illuminate the precise mechanisms and different receptors involved in the effects of the different types of rare ginsenosides, for the subsequent development of safe and nontoxic nutrient supplements for improving sleep and ameliorating insomnia.

\section{Funding}

This work was supported by the National Natural Science Foundation of China (21808184, 21776227, 21878246, 21576223, 21576160); Shaanxi Key Laboratory of Degradable Biomedical Materials Program (18JS105, 15JS106, 2016SZSj-35).

\section{Conflicts of interest}

There are no conflicts of interest to declare.

\section{References}

1 K. Doghramji, The Evaluation and Management of Insomnia, Clin. Chest Med., 2010, 31, 327-339.

2 D. Karl, The epidemiology and diagnosis of insomnia, Am. J. Manag. Care, 2006, 12, 214-220.

$3 \mathrm{H}$. Ashton, Guidelines for the rational use of benzodiazepines. When and what to use, Drugs, 1994, 48, 25-40.

4 G. Chouinard, Issues in the clinical use of benzodiazepines: potency, withdrawal, and rebound, J. Clin. Psychiatry, 2004, 5, 7-12.

5 J. R. Stone, T. S. Zorick and J. Tsuang, Dose-related illusions and hallucinations with zaleplon, Clin. Toxicol., 2009, 46, 344-345.

6 S. Shibata, O. Tanaka, K. Soma, T. Ando, Y. Iida and H. Nakamura, Studies on saponins and sapogenins of ginseng. The structure of panaxatriol, Tetrahedron Lett., 1965, 6, 207-213.

7 L. P. Christensen, Chapter 1 Ginsenosides : Chemistry, Biosynthesis, Analysis, and Potential Health Effects, Adv. Food Nutr. Res., 2009, 55, 1-99.

8 J. H. Song, K. S. Kang and Y. K. Choi, Protective effect of casuarinin against glutamate-induced apoptosis in HT22 cells through inhibition of oxidative stress-mediated MAPK phosphorylation, Bioorg. Med. Chem. Lett., 2017, S0960894X17310740.

9 J. Zhao, S. Lu, H. Yu, S. Duan and J. Zhao, Baicalin and ginsenoside $\mathrm{Rb} 1$ promote the proliferation and differentiation of neural stem cells in Alzheimer's disease model rats, Brain Res., 2017, 1678.

10 S. A. Nag, J. J. Qin, W. Wang, M. H. Wang, H. Wang and R. Zhang, Ginsenosides as Anticancer Agents: In vitro and in vivo Activities, Structure-Activity Relationships, and Molecular Mechanisms of Action, Front. Pharmacol., 2012, $3,25$.
11 L. Seo Young, K. Geun Tae, R. S. Hun, S. Jin-Su, K. Hie-Joon, H. Soon-Sun, K. Sung Won and P. Jeong Hill, Proteomic analysis of the anti-cancer effect of 20S-ginsenoside Rg3 in human colon cancer cell lines, Journal of the Agricultural Chemical Society of Japan, 2009, 73, 811-816.

12 S. Kang, S. J. Park, A. Y. Lee, J. Huang, H. Y. Chung and D. S. Im, Ginsenoside Rg3 promotes inflammation resolution through M2 macrophage polarization, J. Ginseng Res., 2018, 42, 68-74.

13 J. Deng, Y. Liu, Z. Duan, C. Zhu, J. Hui, M. Yu, P. Ma, X. Ma, D. Fan and H. Yang, Protopanaxadiol and ProtopanaxatriolType Saponins Ameliorate Glucose and Lipid Metabolism in Type 2 Diabetes Mellitus in High-Fat Diet/StreptozocinInduced Mice, Front. Pharmacol., 2017, 8, 506.

14 V. Darias, S. Abdala, D. Martin-Herrera, M. L. Tello and S. Vega, CNS effects of a series of 1,2,4-triazolyl heterocarboxylic derivatives, Pharmazie, 1998, 53, 477.

15 C. Wolfman, H. Viola, M. Marder, C. Wasowski, P. Ardenghi, I. Izquierdo, A. C. Paladini and J. H. Medina, Anxioselective properties of 6,3'-dinitroflavone, a high-affinity benzodiazepine receptor ligand, Eur. J. Pharmacol., 1996, 318, 23-30.

16 L. M. Paterson, S. J. Wilson, D. J. Nutt, P. H. Hutson and M. Ivarsson, A translational, caffeine-induced model of onset insomnia in rats and healthy volunteers, Psychopharmacology, 2007, 191, 943-950.

17 C. Zhang, X. Zhao, X. Mao, A. Liu, Z. Liu, X. Li, K. Bi and Y. Jia, Pharmacological evaluation of sedative and hypnotic effects of schizandrin through the modification of pentobarbital-induced sleep behaviors in mice, Eur. J. Pharmacol., 2014, 744, 157-163.

18 A. A. Borbély, H. U. Neuhaus and I. Tobler, Effect of pchlorophenylalanine and tryptophan on sleep, EEG and motor activity in the rat, Behav. Brain Res., 1981, 2, 1-22.

19 Y. Wang, Y. Liu, X. Y. Zhang, L. H. Xu, D. Y. Ouyang, K. P. Liu, H. Pan, J. He and X. H. He, Ginsenoside Rg1 regulates innate immune responses in macrophages through differentially modulating the NF- $\mathrm{BB}$ and PI3K/Akt/ mTOR pathways, Int. Immunopharmacol., 2014, 23, 77-84.

20 X. Jing-Tian, S. Zuo-Hui, T. L. Vanden Hoek, C. Wei-Tien, L. Jing, M. Sangeeta, W. Chong-Zhi, H. Chin-Wang, L. B. Becker and Y. Jun-Jie, Antioxidant effects of ginsenoside Re in cardiomyocytes, Eur. J. Pharmacol., 2006, 532, 201-207.

21 S. Kang, S. J. Park, A. Y. Lee, H. Jin, H. Y. Chung and D. S. Im, Ginsenoside Rg3promotes inflammation resolution through M2 macrophage polarization, J. Ginseng Res., 2018, 42, 6874.

22 Z. Duan, B. Wei, J. Deng, Y. Mi, Y. Dong, C. Zhu, R. Fu, L. Qu and D. Fan, The anti-tumor effect of ginsenoside Rh4 in MCF-7 breast cancer cells invitro and invivo, Biochem. Biophys. Res. Commun., 2018, 499, 482-487.

23 M. Zhu, N. G. Bowery, P. M. Greengrass and J. D. Phillipson, Application of radioligand receptor binding assays in the search for CNS active principles from Chinese medicinal plants, J. Ethnopharmacol., 1996, 54, 153-164. 
24 Y. Öztürk, S. Aydin, R. Beis, K. H. C. Başer and H. Berberoĝlu, Effects of Hypericum perforatum L. and Hypericum calycinum $\mathrm{L}$. extracts on the central nervous system in mice, Phytomedicine, 1996, 3, 139-146.

25 J. K. Wyatt, C. Cajochen, C. A. Ritz-De, C. A. Czeisler and D. J. Dijk, Low-dose repeated caffeine administration for circadian-phase-dependent performance degradation during extended wakefulness, Sleep, 2004, 27, 374-381.

26 H. P. Landolt, D. J. Dijk, S. E. Gaus and A. A. Borbély, Caffeine reduces low-frequency delta activity in the human sleep EEG, Neuropsychopharmacology, 1995, 12, 229-238.

27 P. Y. Yue, D. Y. Wong, P. K. Wu, P. Y. Leung, N. K. Mak, H. W. Yeung, L. Liu, Z. Cai, Z. H. Jiang and T. P. Fan, The angiosuppressive effects of $20(\mathrm{R})$ - $^{-}$ginsenoside Rg3, Biochem. Pharmacol., 2006, 72, 437-445.

28 W. Wang, Y. Zhao, E. R. Rayburn, D. L. Hill, H. Wang and R. Zhang, In vitro anti-cancer activity and structure-activity relationships of natural products isolated from fruits of Panax ginseng, Cancer Chemother. Pharmacol., 2007, 59, 589-601.

29 U. Dougherty, R. Mustafi, Y. Wang, M. W. Musch, C. Z. Wang, V. J. Konda, A. Kulkarni, J. Hart, G. Dawson and K. E. Kim, American ginseng suppresses Western dietpromoted tumorigenesis in model of inflammationassociated colon cancer: role of EGFR, BMC Complementary Altern. Med., 2011, 11, 111.

30 J. M. Monti, Serotonin control of sleep-wake behavior, Sleep Med. Rev., 2011, 15, 269-281.

31 W. J. Mcentee and T. H. Crook, Serotonin, memory, and the aging brain, Psychopharmacology, 1991, 103, 143-149.

32 C. M. Portas, B. Bjorvatn and R. Ursin, Serotonin and the sleep/wake cycle: special emphasis on microdialysis studies, Prog. Neurobiol., 2000, 60, 13-35.

33 J. X. Zhang, S. R. Li, S. Yao, Q. R. Bi, J. J. Hou, L. Y. Cai, S. M. Han, W. Y. Wu and D. A. Guo, Anticonvulsant and sedative-hypnotic activity screening of pearl and nacre (mother of pearl), J. Ethnopharmacol., 2016, 181, 229-235.

34 R. Ursin, Serotonin and sleep, Sleep Med. Rev., 2002, 6, 5567.

35 X. Zhao, X. Y. Cui, B. Q. Chen, Q. P. Chu, H. Y. Yao, B. S. Ku and Y. H. Zhang, Tetrandrine, a bisbenzylisoquinoline alkaloid from Chinese herb Radix, augmented the hypnotic effect of pentobarbital through serotonergic system, Eur. J. Pharmacol., 2004, 506, 101-105.

36 Y. H. Zhang and L. H. Fang, Antagonism of morphineinduced antinociception by tetrandrine is dependent on serotonergic mechanisms, Life Sci., 2001, 69, 1429-1439.

37 C. Dugovic, Role of serotonin in sleep mechanisms, Rev. Neurol., 2001, 157, 16-19.

38 A. Khateb, P. Fort, A. Alonso, B. E. Jones and M. Mülethaler, Pharmacological and Immunohistochemical Evidence for Serotonergic Modulation of Cholinergic Nucleus Basalis Neurons, Eur. J. Neurosci., 1993, 5, 541-547.

39 L. Levita, S. E. Hammack, I. Mania, X. Y. Li, M. Davis and D. G. Rainnie, 5-hydroxytryptamine1A-like receptor activation in the bed nucleus of the stria terminalis: electrophysiological and behavioral studies, Neuroscience, 2004, 128, 583-596.

40 I. Mody, Y. D. Koninck, T. S. Otis and I. Soltesz, Bridging the cleft at GABA synapses in the brain, Trends Neurosci., 1994, $17,517$.

41 A. N. Bateson, The benzodiazepine site of the GABAA receptor: an old target with new potential?, Sleep Med., 2004, 5, S9-S15.

42 C. R. Chen, X. Z. Zhou, Y. J. Luo, Z. L. Huang, Y. Urade and W. M. Qu, Magnolol, a major bioactive constituent of the bark of Magnolia officinalis, induces sleep via the benzodiazepine site of $\operatorname{GABA}(\mathrm{A})$ receptor in mice, Neuropharmacology, 2012, 63, 1191-1199. 